\title{
Article
}

\section{Predictor Analysis in Group Decision Making}

\author{
Stan Lipovetsky $\mathbb{1}$
}

check for updates

Citation: Lipovetsky, S. Predictor Analysis in Group Decision Making. Stats 2021, 4, 108-121. https:// doi.org/10.3390/stats4010009

Received: 13 January 2021

Accepted: 4 February 2021

Published: 9 February 2021

Publisher's Note: MDPI stays neutral with regard to jurisdictional claims in published maps and institutional affiliations.

Copyright: (C) 2021 by the author. Licensee MDPI, Basel, Switzerland. This article is an open access article distributed under the terms and conditions of the Creative Commons Attribution (CC BY) license (https:// creativecommons.org/licenses/by/ $4.0 /)$.
Independent Researcher, 13417 Inverness Rd., Minnetonka, Minneapolis, MN 55305, USA; stan.lipovetsky@gmail.com

\begin{abstract}
Priority vectors in the Analytic Hierarchy Process (AHP) are commonly estimated as constant values calculated by the pairwise comparison ratios elicited from an expert. For multiple experts, or panel data, or other data with varied characteristics of measurements, the priority vectors can be built as functions of the auxiliary predictors. For example, in multi-person decision making, the priorities can be obtained in regression modeling by the demographic and socio-economic properties. Then the priorities can be predicted for individual respondents, profiled by each predictor, forecasted in time, studied by the predictor importance, and estimated by the characteristic of significance, fit and quality well-known in regression modeling. Numerical results show that the suggested approaches reveal useful features of priority behavior, that can noticeably extend the AHP abilities and applications for numerous multiple-criteria decision making problems. The considered methods are useful for segmentation of the respondents and finding optimum managerial solutions specific for each segment. It can help to decision makers to focus on the respondents' individual features and to increase customer satisfaction, their retention and loyalty to the promoted brands or products.
\end{abstract}

Keywords: AHP priority as functions; exponential and multinomial models; auxiliary predictors

\section{Introduction}

Analytic Hierarchy Process (AHP) is one of the main methods for solving various multiple-criteria decision making problems. It had been originated by Thomas Saaty [1-5] and developed in works of many authors [6-13]. The preferences in the AHP can be calculated by different techniques using the pairwise comparison matrices to find the vectors of priorities presented as constant values.

However, for multiple experts, or panel data, or other problems with multiple matrices of paired comparisons elicited in different conditions with varied characteristics of measurements, the priority vectors can be built not as constants but as functions of some additional auxiliary predictors. For example, in multi-person decision making applied in marketing, advertising, and other applied social research, performed by means of the AHP or any other tool of statistical modeling, the information on the respondents' demographics and other characteristics of the economics environment is always gathered. There are research problems with AHP pairwise priorities elicited, for example, from the side of managers or the side of customers at different store locations, where many dozens of respondents were questioned.

Pair comparison techniques operate with multiple values, for example, of the AHP pairwise ratios, which are used for evaluation of the final priorities and the corresponding ranks of the preferences. A simple asking respondents to rank order the multiple items is not so easy task because it requires to hold in mind all the items simultaneously. In contrast, the pair comparisons approach, when a respondent should compare only two of the presented items, yield more reliable relations between these items' preferences in the whole set of them. The pairwise ratios are elicited from a respondent for whom we can know the demographic variables, and to use them to a better fit and better prediction of the priorities. The preferences evaluated without auxiliary variables correspond to 
regressions without the predictors-those produce simply the constant mean value of the elicited preferences.

The current work explores new approaches for constructing the priority vectors with elements presented by the functions found via regression modeling. The varying priorities can be predicted for individual respondents, profiled by each predictor, forecasted in time, studied by the predictor importance, and estimated by the characteristic of significance and quality known in the regression modeling. Numerical results show that the suggested approaches reveal useful features of priority behavior that can noticeably extend the AHP abilities and applications for numerous multiple-criteria decision making problems.

In the proposed approach, the priorities of the compared items can be found not only on the aggregated level, but for each individual respondent based on her/his tastes, views, perceptions, attitudes, predispositions, and any other characteristics and preferences. It can help to decision makers and managers to focus on the respondents' individual needs and to elaborate special approaches to increase customer satisfaction, their retention and loyalty to the suggested brands or products. The considered methods are also useful for segmentation of the respondents and finding optimum managerial solutions specific for each segment. In regression analysis, a possibility of finding personalized key drivers for individual respondents was studied in [14].

The paper is organized as follows: Section 2 describes AHP priority estimations as constant parameters, and Section 3 extends the priorities to be expressed as functions by the predictors. Section 4 discusses numerical examples, and Section 5 summarizes.

\section{AHP Priority Estimated via Minimization of Deviations}

Priority estimation in the classical AHP is performed by the matrix of pairwise judgements elicited from an expert. Each element $a_{j k}$ of this matrix shows a quotient of preference of the $j$-th item over $k$-th item in their comparison among all $n$ items. The elements of this matrix are reciprocally symmetrical,

$$
a_{j k}=1 / a_{k j} .
$$

A theoretical Saaty matrix of pair comparisons defines each $j k$-th element as a ratio of the unknown priorities $w_{j}$ and $w_{k}$ :

$$
W=w *(1 / w)^{\prime}
$$

where the theoretical priorities presented by the vector-column $w$ consists of the elements $w_{1}, w_{2}, \ldots, w_{n}$, the vector-row $(1 / w)^{\prime}$ contains the reciprocal values $1 / w_{1}, 1 / w_{2}, \ldots, 1 / w_{n}$, and the matrix (2) is defined by their outer product (the prime denotes transposition). In a hypothetical case of the perfectly elicited paired ratios coinciding with the theoretical quotients of priorities we could equalize the values from the matrices (1) and (2):

$$
a_{j k}=w_{j} / w_{k} .
$$

However, in practical observations, there is no such perfect data, and the relations (3) should be described in some models with deviations from the exact equalities. For example, assuming a multiplicative error term, the relation (3) can be represented as follows:

$$
a_{j k}=\frac{\alpha_{j}}{\alpha_{k}}\left(1+\delta_{j k}\right),
$$

where alpha $\alpha_{j}$ denotes the estimates for the elements of the theoretical priority vector $w$, and delta $\delta_{j k}$ denotes the relative errors.

The relations (4) can be represented in a linear form by multiplying by the denominator $\alpha_{k}$,

$$
a_{j k} \alpha_{k}=\alpha_{j}\left(1+\delta_{j k}\right) .
$$


Summing by $k$ yields the Equations:

$$
\sum_{k=1}^{n} a_{j k} \alpha_{k}=\alpha_{j}\left(n+\sum_{k=1}^{n} \delta_{j k}\right) .
$$

Equalizing the sums of errors at the right-hand side (6) for all $j$ and denoting them as

$$
\lambda=n+\sum_{k=1}^{n} \delta_{j k}
$$

we can rewrite the Equation (6) in the matrix form as follows:

$$
A \alpha=\lambda \alpha .
$$

The expression (8) is the AHP eigenproblem for the matrix $A$ with elements (1), and its eigenvector $\alpha$ for the maximum eigenvalue lambda (7) defines the vector of priorities. The problem (8) is usually obtained by analogue with the relation $W w=n w$ for the theoretical matrix and vector (2), but the derivation (4)-(8) shows explicitly that the classical AHP corresponds to equalizing the total relative deviations in the rows of the matrix $A$, and the mean level of these totals is close to the number of compared alternatives $n$ (7).

Linearizing the relations (4) can be done by logarithmic transformation as well:

$$
\ln a_{j k}=\ln \alpha_{j}-\ln \alpha_{k}+\ln \left(1+\delta_{j k}\right) .
$$

Then the Least Squares (LS) minimization for the relative deviations can be applied:

$$
L S=\sum_{j, k=1}^{n}\left(\ln \left(1+\delta_{j k}\right)\right)^{2}=\sum_{j, k=1}^{n}\left(\ln a_{j k}-\ln \alpha_{j}+\ln \alpha_{k}\right)^{2} .
$$

For small relative deviations $\left|\delta_{j k}\right| \ll 1$, the decomposition $\ln \left(1+\delta_{j k}\right)=\delta_{j k}$ shows that the expression at the left-hand side (10) has a meaning of the relative errors squared. Minimizing this objective by the logarithm of estimated priorities $\ln \alpha_{j}$ produces the logarithmic least squares, also known as multiplicative solution with priorities presented by the geometric means in the rows of matrix $A(1)$.

Instead of the relative errors in (4), it is possible to take a model with absolute errors:

$$
a_{j k}=\frac{\alpha_{j}}{\alpha_{k}}+\varepsilon_{j k}
$$

where $\varepsilon_{j k}$ are the deviations between the elicited values in (1) and their approximation via the priority estimates alpha $\alpha_{j}$. The LS for the absolute errors corresponds to the objective

$$
L S=\sum_{j, k=1}^{n} \varepsilon_{j k}^{2}=\sum_{j, k=1}^{n}\left(a_{j k}-\frac{\alpha_{j}}{\alpha_{k}}\right)^{2} .
$$

The priorities can be found in the nonlinear LS estimation for this criterion. A linearization similar to the transformation (4) to Equation (5) can be applied to the model (11) with absolute errors, leading to the linear least square estimation for the priority vector. An obtained priority vector is usually standardized in AHP by the total of its elements:

$$
p_{j}=\frac{\alpha_{j}}{\sum_{k=1}^{n} \alpha_{k}},
$$

so that the sum of the normalized components equals one. More details on all these and other solutions can be found in $[6,11,13,15,16]$. 


\section{AHP Priority Vectors Modeled by Predictors}

The more complicated problems of group decision making include multiple experts whose opinions can vary because of their demographic, socio-economics, and many other functional characteristics impacting their pairwise ratios' evaluations. Some other problems can contain panel data where observations are elicited in different moments in time, or for varying prices, so the preferences among the alternative can depend on the changing conditions. Such more complicated situations require the above considered models to be extended to incorporating auxiliary variables explaining variability of the priorities across the respondents and their specific features. Let us describe several possible ways to expand the common AHP techniques to more sophisticated methods which permit to account for auxiliary variables in the preference modeling.

The theoretical priorities $w_{j}$ in (2) can be described as the exponent functions:

$$
w_{j}^{i}=\exp \left(w_{j 0}+w_{j 1} x_{1}^{i}+w_{j 2} x_{2}^{i}+\cdots+w_{j M} x_{M}^{i}\right),
$$

where $x_{1}^{i}, x_{2}^{i}, \cdots x_{M}^{i}$ are the auxiliary predictors (with the subscript $m=1,2, \ldots, M-$ number of the variables; and the superscript $i=1,2, \ldots, N$-number of the respondents); the notations $W_{j 0}, W_{j 1}, W_{j 2}, \ldots, W_{j M}$ stand for the parameters of the $j$-th theoretical priority expressed via the intercept and the coefficients for linear combination of predictors. The exponents guarantee a positive total priority $W_{j}^{i}$ of $j$-th alternative for $i$-th respondent at the left-hand side (14) for any set of the predictors' values.

Consider the model (4) with the odds built by the exponents (14), but in place of all theoretical parameters $w$ we use their estimates denoted by the corresponding parameters alpha. The constant parameters of preference $\alpha_{j}$ and $\alpha_{k}$ we extend by substitution with the exponential functions:

$$
a_{j k}^{i}=\frac{\alpha_{j}^{i}}{\alpha_{k}^{i}}\left(1+\delta_{j k}^{i}\right)=\frac{\exp \left(\alpha_{j 0}+\alpha_{j 1} x_{1}^{i}+\alpha_{j 2} x_{2}^{i}+\cdots+\alpha_{j M} x_{M}^{i}\right)}{\exp \left(\alpha_{k 0}+\alpha_{k 1} x_{1}^{i}+\alpha_{k 2} x_{2}^{i}+\cdots+\alpha_{k M} x_{M}^{i}\right)}\left(1+\delta_{j k}^{i}\right) .
$$

The pairwise ratios $a_{j k}^{i}$ and deviations $\delta_{j k}^{i}$ correspond to the comparison of $j$-th and $k$-th alternatives by the $i$-th respondent, and all other notations are the same as in the expression (14), subject to substitution of theoretical parameters $w$ by their estimates as alpha parameters. In absence of the auxiliary $x$-variables, the model (15) reduces to the model (4) with the notations $a_{j}=\exp \left(a_{j 0}\right)$, averaged across the respondents.

Applying to (15) the logarithmic transformation (9) and LS criterion (10) yields:

$$
\begin{gathered}
L S=\sum_{i=1}^{N} \sum_{j, k=1}^{n}\left(\ln \left(1+\delta_{j k}^{i}\right)\right)^{2} \\
=\sum_{i=1}^{N} \sum_{j, k=1}^{n}\left(\ln a_{j k}^{i}-\left(\alpha_{j 0}-\alpha_{k 0}\right)-\left(\alpha_{j 1}-\alpha_{k 1}\right) x_{1}^{i}-\cdots-\left(\alpha_{j M}-\alpha_{k M}\right) x_{M}^{i}\right)^{2} .
\end{gathered}
$$

Instead of the odds ratio model (15)-(16) with the relative errors, we can consider the model (11), (12) with the absolute errors as well.

Another approach based not on the pairwise ratios but directly on the preference shares in total (13) can be built using the multinomial-logit model expressed via the exponents (14):

$$
p_{j}^{i}=\frac{w_{j}^{i}}{\sum_{k=1}^{i} w_{k}^{i}}=\frac{\exp \left(w_{j 0}+w_{j 1} x_{1}^{i}+w_{j 2} x_{2}^{i}+\cdots+w_{j M} x_{M}^{i}\right)}{\sum_{k=1}^{n} \exp \left(w_{k 0}+w_{k 1} x_{1}^{i}+w_{k 2} x_{2}^{i}+\cdots+w_{k M} x_{M}^{i}\right)} .
$$

For an $i$-th respondent in (17), the shares of $w_{j}$ in their total can be evaluated by the pairwise comparison matrix (2) as follows:

$$
\frac{w_{j}}{\sum_{k=1}^{i} w_{k}}=\frac{1}{\sum_{k=1}^{n} w_{k} / w_{j}}=\frac{1}{\sum_{k=1}^{n} w_{k j}}=\frac{1}{\sum_{k=1}^{n} 1 / W_{j k}}=H_{j} / n
$$


That is the reciprocal of totals in each $j$-th column of the theoretical matrix $W$, which is proportional to the harmonic means $H_{j}$ of the elements in each $j$-th row of the matrix (2). For the elicited pairwise ratios in matrix (1), such values correspond to the inverted totals in each column of the matrix $A^{i}$, or the inverted totals of the reciprocals in its each row, $1 / \sum_{k=1}^{n} 1 / A_{j k}^{i}$, where the superscript $i$ identifies the matrix of the pairwise ratios for the $i$-th respondent. Using these values in (17) and rewriting it as the model with relative errors expressed via the estimate alpha in place of the theoretical preferences $w$, yields the following expression:

$$
\frac{1}{\sum_{k=1}^{n} 1 / A_{j k}^{i}}=\frac{\exp \left(\alpha_{j 0}+\alpha_{j 1} x_{1}^{i}+\alpha_{j 2} x_{2}^{i}+\cdots+\alpha_{j M} x_{M}^{i}\right)}{\sum_{k=1}^{n} \exp \left(\alpha_{k 0}+\alpha_{k 1} x_{1}^{i}+\alpha_{k 2} x_{2}^{i}+\cdots+\alpha_{k M} x_{M}^{i}\right)}\left(1+\delta_{j k}^{i}\right) .
$$

Then similarly to (16), it is easy to build the LS criterion for minimization of the relative deviations in (19).

For uniqueness of solution in finding parameters of the multinomial-logit model (19), one set of the coefficients is commonly put to zero, for instance, the first share's parameters equal zero. The same approach can be used in the modeling (14)-(16) with ratios of the exponent odds.

Let us make several additional remarks useful for numerical modeling. Instead of the relative errors, the models with absolute errors similar to (11), (12) can be tried for the odds (14)-(16) or the shares (17)-(19) models too. In place of the considered LS criterion, the maximum likelihood criterion is often used for parameter estimation in the multinomiallogit modeling. Sometimes the general multinomial models are simplified to the so-called conditional logits which use the same parameters for all shares and differ only by the predictors' values. All these models are widely applied in the choice modeling [17-19]. For all pairwise ratios elicited from a respondent, the demographic or other auxiliary values are the same, so the described methods correspond to the multi-person decision making as employed in [12]. The non-linear estimations needed for several considered approaches can be performed with the modern statistical packages, for example, [20-23]. It is useful to mention that the predictions by the ordinary least squares regressions produce results not depending on the degree of multicollinearity. However, for analysis of the individual parameters of the model, the regressions can be constructed not only by LS criterion as in (16), but by special techniques helpful for evaluation of the predictors' importance and parameters of models not prone to the effects of multicollinearity. Detail description of these methods can be found, for example, in [24,25].

In the described models, we find parameters of the priority presentation via the predictors, then we can predict values of the priorities themselves. A simpler way to reach this goal is also possible with multiple respondents to find individual priorities of the alternatives for each respondent, then to model them by the demographics and other auxiliary predictors. Suppose, the priorities for each respondent are found by a method considered in (1)-(13), and they are stacked with the individual demographic characteristics. Then we can build a set of multiple regressions of each $j$-th alternative in its dependence on the predictors and make predictions by $i$-th respondents:

$$
\alpha_{j}^{i}=\alpha_{j 0}+\alpha_{j 1} x_{1}^{i}+\alpha_{j 2} x_{2}^{i}+\cdots+\alpha_{j M} x_{M}^{i}
$$

To be sure that the predicted values of the priorities are always positive, it is preferable to apply exponential functions like those used in (14)-(19):

$$
\alpha_{j}^{i}=\exp \left(\alpha_{j 0}+\alpha_{j 1} x_{1}^{i}+\alpha_{j 2} x_{2}^{i}+\cdots+\alpha_{j M} x_{M}^{i}\right)
$$

By the logarithm transformation, the relations (21) can be reduced to the linear regressions which are more convenient in practical modeling. 


\section{Numerical Comparisons for Priority Estimations}

Data from a marketing research project is taken on 17 respondents who evaluated eight flavors of a snack. The flavors are denoted and called as follows: A-Gratin, B-Sweet, CCream, D-Gold, E-Rosemary, F-Butter, G-Chives, H-Cheese. The last six columns in Table 1 contain demographic data of the respondents: Gender (1-male, 2-female), Age groups (1—under age $18 ; 2-18: 20$ years old; $3-21: 24 ; 4-25: 29 ; 5-30: 34 ; 6-35: 39$; 7-40:44; 8-45:49; 9-50:54; 10—55:59; 11—60:64; and 12-65 or older), HH-how many people are in the household (1-one person, 2-two people, 3-three, 4-four, 5-five or more), Education (1-grade school or less; 2-some high school; 3 - high school graduate; 4-some college; 5-two-year college or technical school; 6-four-year college; 7-some postgraduate work; 8 - postgraduate degree), Employment (1-work full-time; 2-work part-time; 3-self-employed; 4-student; 5-homemaker; 6-Retired), Income group, in thousand \$ (1—less than 25; 2-25:39; 3-40:49; 4-50:74; 5—75:99; 6-100:124; 7-125:149; 8 -150 or more).

Table 1. Initial data on AHP pairwise ratios and demographics.

\begin{tabular}{|c|c|c|c|c|c|c|c|c|c|}
\hline ID & $a_{12}$ & $a_{13}$ & $a_{23}$ & Gender & Age & Household & Education & Employment & Income \\
\hline 1 & 2 & 0.5 & 0.25 & 2 & 8 & 4 & 3 & 5 & 2 \\
\hline 2 & 4 & 1 & 0.25 & 2 & 4 & 2 & 4 & 5 & 2 \\
\hline 3 & 2 & 0.5 & 0.333333 & 2 & 5 & 3 & 6 & 1 & 4 \\
\hline 4 & 0.5 & 1 & 1 & 2 & 4 & 2 & 7 & 1 & 2 \\
\hline 5 & 1 & 1 & 1 & 1 & 6 & 1 & 3 & 3 & 2 \\
\hline 6 & 2 & 1 & 1 & 2 & 10 & 2 & 3 & 5 & 3 \\
\hline 7 & 0.5 & 0.125 & 0.25 & 1 & 10 & 2 & 5 & 6 & 2 \\
\hline 8 & 4 & 1 & 0.333333 & 2 & 8 & 3 & 6 & 5 & 5 \\
\hline 9 & 6 & 1 & 0.2 & 2 & 9 & 2 & 3 & 5 & 5 \\
\hline 10 & 0.2 & 0.333333 & 2 & 1 & 5 & 1 & 4 & 1 & 1 \\
\hline 11 & 3 & 0.5 & 0.2 & 1 & 12 & 2 & 6 & 6 & 3 \\
\hline 12 & 2 & 1 & 0.25 & 2 & 10 & 2 & 4 & 6 & 2 \\
\hline 13 & 3 & 1 & 0.25 & 1 & 10 & 3 & 4 & 3 & 8 \\
\hline 14 & 1 & 1 & 0.5 & 1 & 6 & 5 & 8 & 1 & 3 \\
\hline 15 & 8 & 1 & 0.142857 & 1 & 8 & 1 & 6 & 1 & 4 \\
\hline 16 & 4 & 1 & 0.333333 & 1 & 5 & 3 & 3 & 1 & 6 \\
\hline 17 & 0.5 & 0.5 & 1 & 1 & 7 & 2 & 3 & 1 & 1 \\
\hline
\end{tabular}

For three flavors (1-A, 2-E, and 3-H) of the main interest, the AHP elicited pairwise comparison ratios and the demographic characteristics of the respondents are presented in Table 1.

The three pairwise quotients in the first columns of Table 1 correspond to the elements $a_{12}, a_{13}$, and $a_{23}$ in the AHP matrices. For instance, the 1st and 15th respondents' matrices are:

$$
A_{1}=\left(\begin{array}{ccc}
1 & 2 & 1 / 2 \\
1 / 2 & 1 & 1 / 4 \\
2 & 4 & 1
\end{array}\right), A_{15}=\left(\begin{array}{ccc}
1 & 8 & 1 \\
1 / 8 & 1 & 1 / 7 \\
1 & 7 & 1
\end{array}\right)
$$

where we take into account that there are 1s on the diagonal, and due to (1) the lower triangle elements are reciprocal to the upper triangle elements.

The initial data from Table 1 are represented in the extended-by-row matrix which is more convenient for the regressions, as it is often used in the discrete choice modeling. For this aim, each row of Table 1 is presented in three rows, by the number of the outcome variables of pair ratios, and a new dependent variable can be denoted as $y_{v}$ which takes all the values $a_{j k}$. For $n$ compared alternatives, there are $n(n-1) / 2$ resulting paired quotients, so with $N$ respondents the total number of observations becomes:

$$
V=N \cdot n(n-1) / 2 \text {. }
$$


In our example with 3 items and 17 respondents, the total number (23) of the observed values is 51 , with so many values $y_{v}$ (where $v=1,2, \ldots, V$ ). Therefore, not the number of respondents, but the number of the responses defines the total number of observations.

Table 2 presents such an extended matrix for regression modeling-let us describe it in more detail. After the columns of ID for 17 respondents and 51 of the observations number $v$, there are two columns of binary indicators $\mathrm{A}$ and $\mathrm{H}$, where $\mathrm{A}$ indicates by 1 that this item is in the numerator of the paired ratio, and by 0 that $\mathrm{A}$ is absent in the paired ratio; similarly, in $\mathrm{H}$-zero means that it is not in the current pair comparison, and -1 if this item takes place in the denominator of the paired ratio. The column for the $\mathrm{E}$ item is excluded as redundant-the total of the three columns A, E, and $\mathrm{H}$ equals zero identically, so one of them is not needed for modeling. As it was mentioned after the formula (19), one set of the parameters can be put to zero for the uniqueness of solution for the regression, and it corresponds to exclusion of the redundant data. Instead of the column for E item, we could exclude the item $\mathrm{A}$, or the item $\mathrm{H}$-the results of the regression modeling would be with other parameters but produce the same predictions.

Besides the binary variables $\mathrm{A}$ and $\mathrm{H}$ for the modeling (14)-(16), we also take the demographic auxiliary predictors in two sets-one related to the item A and another one to the item $\mathrm{H}$, as in the numerator and denominator in the ratios (15). The predictors related to the item A are taken with positive or zero values exactly in correspondence with the values in the column A indicating if this item is presented in the current paired ratio numerator or not. Similarly, the predictors related to the item $\mathrm{H}$ are taken with negative or zero values in correspondence with the values in the column $\mathrm{H}$ indicating if this item is presented in the current paired ratio denominator or not. In Table 2, the set of the demographic variables which goes with the item A is denoted by the share number 1 (for example, gender.1, age.1, $\ldots$, income.1), and the set which goes with the item $\mathrm{H}$ is denoted by the share number 3 . The set for the share related to the second item $E$ is excluded as redundant by the same reason of getting a unique solution, as discussed above.

For $n$ alternatives, a combined model has $n-1$ sets of parameters, and with $M$ auxiliary predictors there are $M+1$ parameters for each set, so total $(n-1) \cdot(M+1)$ parameters (plus a general intercept which is usually insignificant and can be omitted). For building a regression, the total number of observations (23) should be bigger than the total number of parameters, so approximately such an inequality should hold:

$$
N \cdot n / 2>M+1
$$

In the example with $n=3, N=17$, and $M=6$, this inequality is as $25>7$, that is acceptable for a regression modeling where it is advised to have at least three time more observations than parameters.

Using logarithms of the ratio values as functions by all predictors in the design in Table 2, we build the linearized model with all shares' parameters obtained simultaneously in one regression. The results of this modeling are presented in Table 3. In the first numerical column, there are estimates for the parameters of the priorities $\mathrm{A}$ and $\mathrm{H}$, and the complementary parameters on the demographics related to these first and third shares. The next are columns of the standard errors, t-statistics, and p-values of the coefficients. We see that mostly the Income predictors are significant in the model for both shares. Below the parameters, there are characteristics of the model quality: coefficient of multiple determination $R^{2}=0.6877$, with the related $F$-statistics on 14 and 36 degrees of freedom, and its $p$-value — those are good and tell us that the whole model is acceptable. 
Table 2. AHP and demographic data arranged for regression modeling.

\begin{tabular}{|c|c|c|c|c|c|c|c|c|c|c|c|c|c|c|c|c|}
\hline ID & obs. $v$ & $\begin{array}{c}\text { Ratio } \\
y_{v}\end{array}$ & A & $\mathbf{H}$ & Gender.1 & Age.1 & Household.1 & Education.1 & Employment.1 & Income.1 & Gender.3 & Age.3 & Household.3 & Education.3 & Employment.3 & Income.3 \\
\hline 1 & 1 & 2 & 1 & 0 & 2 & 8 & 4 & 3 & 5 & 2 & 0 & 0 & 0 & 0 & 0 & 0 \\
\hline 1 & 2 & 0.5 & 1 & -1 & 2 & 8 & 4 & 3 & 5 & 2 & -2 & -8 & -4 & -3 & -5 & -2 \\
\hline 1 & 3 & 0.25 & 0 & -1 & 0 & 0 & 0 & 0 & 0 & 0 & -2 & -8 & -4 & -3 & -5 & -2 \\
\hline 2 & 4 & 4 & 1 & 0 & 2 & 4 & 2 & 4 & 5 & 2 & 0 & 0 & 0 & 0 & 0 & 0 \\
\hline 2 & 5 & 1 & 1 & -1 & 2 & 4 & 2 & 4 & 5 & 2 & -2 & -4 & -2 & -4 & -5 & -2 \\
\hline 2 & 6 & 0.25 & 0 & -1 & 0 & 0 & 0 & 0 & 0 & 0 & -2 & -4 & -2 & -4 & -5 & -2 \\
\hline 3 & 7 & 2 & 1 & 0 & 2 & 5 & 3 & 6 & 1 & 4 & 0 & 0 & 0 & 0 & 0 & 0 \\
\hline 3 & 8 & 0.5 & 1 & -1 & 2 & 5 & 3 & 6 & 1 & 4 & -2 & -5 & -3 & -6 & -1 & -4 \\
\hline 3 & 9 & 0.333333 & 0 & -1 & 0 & 0 & 0 & 0 & 0 & 0 & -2 & -5 & -3 & -6 & -1 & -4 \\
\hline 4 & 10 & 0.5 & 1 & 0 & 2 & 4 & 2 & 7 & 1 & 2 & 0 & 0 & 0 & 0 & 0 & 0 \\
\hline 4 & 11 & 1 & 1 & -1 & 2 & 4 & 2 & 7 & 1 & 2 & -2 & -4 & -2 & -7 & -1 & -2 \\
\hline 4 & 12 & 1 & 0 & -1 & 0 & 0 & 0 & 0 & 0 & 0 & -2 & -4 & -2 & -7 & -1 & -2 \\
\hline 5 & 13 & 1 & 1 & 0 & 1 & 6 & 1 & 3 & 3 & 2 & 0 & 0 & 0 & 0 & 0 & 0 \\
\hline 5 & 14 & 1 & 1 & -1 & 1 & 6 & 1 & 3 & 3 & 2 & -1 & -6 & -1 & -3 & -3 & -2 \\
\hline 5 & 15 & 1 & 0 & -1 & 0 & 0 & 0 & 0 & 0 & 0 & -1 & -6 & -1 & -3 & -3 & -2 \\
\hline 6 & 16 & 2 & 1 & 0 & 2 & 10 & 2 & 3 & 5 & 3 & 0 & 0 & 0 & 0 & 0 & 0 \\
\hline 6 & 17 & 1 & 1 & -1 & 2 & 10 & 2 & 3 & 5 & 3 & -2 & -10 & -2 & -3 & -5 & -3 \\
\hline 6 & 18 & 1 & 0 & -1 & 0 & 0 & 0 & 0 & 0 & 0 & -2 & -10 & -2 & -3 & -5 & -3 \\
\hline 7 & 19 & 0.5 & 1 & 0 & 1 & 10 & 2 & 5 & 6 & 2 & 0 & 0 & 0 & 0 & 0 & 0 \\
\hline 7 & 20 & 0.125 & 1 & -1 & 1 & 10 & 2 & 5 & 6 & 2 & -1 & -10 & -2 & -5 & -6 & -2 \\
\hline 8 & 22 & 4 & 1 & 0 & 2 & 8 & 3 & 6 & 5 & 5 & 0 & 0 & 0 & 0 & 0 & 0 \\
\hline 8 & 23 & 1 & 1 & -1 & 2 & 8 & 3 & 6 & 5 & 5 & -2 & -8 & -3 & -6 & -5 & -5 \\
\hline 8 & 24 & 0.333333 & 0 & -1 & 0 & 0 & 0 & 0 & 0 & 0 & -2 & -8 & -3 & -6 & -5 & -5 \\
\hline 9 & 25 & 6 & 1 & 0 & 2 & 9 & 2 & 3 & 5 & 5 & 0 & 0 & 0 & 0 & 0 & 0 \\
\hline 9 & 26 & 1 & 1 & -1 & 2 & 9 & 2 & 3 & 5 & 5 & -2 & -9 & -2 & -3 & -5 & -5 \\
\hline 9 & 27 & 0.2 & 0 & -1 & 0 & 0 & 0 & 0 & 0 & 0 & -2 & -9 & -2 & -3 & -5 & -5 \\
\hline 10 & 28 & 0.2 & 1 & 0 & 1 & 5 & 1 & 4 & 1 & 1 & 0 & 0 & 0 & 0 & 0 & 0 \\
\hline 10 & 29 & 0.333333 & 1 & -1 & 1 & 5 & 1 & 4 & 1 & 1 & -1 & -5 & -1 & -4 & -1 & -1 \\
\hline 10 & 30 & 2 & 0 & -1 & 0 & 0 & 0 & 0 & 0 & 0 & -1 & -5 & -1 & -4 & -1 & -1 \\
\hline 11 & 31 & 3 & 1 & 0 & 1 & 12 & 2 & 6 & 6 & 3 & 0 & 0 & 0 & 0 & 0 & 0 \\
\hline 11 & 32 & 0.5 & 1 & -1 & 1 & 12 & 2 & 6 & 6 & 3 & -1 & -12 & -2 & -6 & -6 & -3 \\
\hline 11 & 33 & 0.2 & 0 & -1 & 0 & 0 & 0 & 0 & 0 & 0 & -1 & -12 & -2 & -6 & -6 & -3 \\
\hline 12 & 34 & 2 & 1 & 0 & 2 & 10 & 2 & 4 & 6 & 2 & 0 & 0 & 0 & 0 & 0 & 0 \\
\hline 12 & 35 & 1 & 1 & -1 & 2 & 10 & 2 & 4 & 6 & 2 & -2 & -10 & -2 & -4 & -6 & -2 \\
\hline 12 & 36 & 0.25 & 0 & -1 & 0 & 0 & 0 & 0 & 0 & 0 & -2 & -10 & -2 & -4 & -6 & -2 \\
\hline 13 & 37 & 3 & 1 & 0 & 1 & 10 & 3 & 4 & 3 & 8 & 0 & 0 & 0 & 0 & 0 & 0 \\
\hline 13 & 38 & 1 & 1 & -1 & 1 & 10 & 3 & 4 & 3 & 8 & -1 & -10 & -3 & -4 & -3 & -8 \\
\hline 13 & 39 & 0.25 & 0 & -1 & 0 & 0 & 0 & 0 & 0 & 0 & -1 & -10 & -3 & -4 & -3 & -8 \\
\hline
\end{tabular}


Table 2. Cont.

\begin{tabular}{|c|c|c|c|c|c|c|c|c|c|c|c|c|c|c|c|c|}
\hline ID & obs. $v$ & $\begin{array}{c}\text { Ratio } \\
y_{v}\end{array}$ & A & $\mathbf{H}$ & Gender.1 & Age.1 & Household.1 & Education.1 & Employment.1 & Income.1 & Gender.3 & Age.3 & Household.3 & Education.3 & Employment.3 & Income.3 \\
\hline 14 & 40 & 1 & 1 & 0 & 1 & 6 & 5 & 8 & 1 & 3 & 0 & 0 & 0 & 0 & 0 & 0 \\
\hline 14 & 41 & 1 & 1 & -1 & 1 & 6 & 5 & 8 & 1 & 3 & -1 & -6 & -5 & -8 & -1 & -3 \\
\hline 14 & 42 & 0.5 & 0 & -1 & 0 & 0 & 0 & 0 & 0 & 0 & -1 & -6 & -5 & -8 & -1 & -3 \\
\hline 15 & 43 & 8 & 1 & 0 & 1 & 8 & 1 & 6 & 1 & 4 & 0 & 0 & 0 & 0 & 0 & 0 \\
\hline 15 & 44 & 1 & 1 & -1 & 1 & 8 & 1 & 6 & 1 & 4 & -1 & -8 & -1 & -6 & -1 & -4 \\
\hline 15 & 45 & 0.142857 & 0 & -1 & 0 & 0 & 0 & 0 & 0 & 0 & -1 & -8 & -1 & -6 & -1 & -4 \\
\hline 16 & 46 & 4 & 1 & 0 & 1 & 5 & 3 & 3 & 1 & 6 & 0 & 0 & 0 & 0 & 0 & 0 \\
\hline 16 & 47 & 1 & 1 & -1 & 1 & 5 & 3 & 3 & 1 & 6 & -1 & -5 & -3 & -3 & -1 & -6 \\
\hline 16 & 48 & 0.333333 & 0 & -1 & 0 & 0 & 0 & 0 & 0 & 0 & -1 & -5 & -3 & -3 & -1 & -6 \\
\hline 17 & 49 & 0.5 & 1 & 0 & 1 & 7 & 2 & 3 & 1 & 1 & 0 & 0 & 0 & 0 & 0 & 0 \\
\hline 17 & 50 & 0.5 & 1 & -1 & 1 & 7 & 2 & 3 & 1 & 1 & -1 & -7 & -2 & -3 & -1 & -1 \\
\hline 17 & 51 & 1 & 0 & -1 & 0 & 0 & 0 & 0 & 0 & 0 & -1 & -7 & -2 & -3 & -1 & -1 \\
\hline
\end{tabular}


Table 3. Model of regression for AHP priority with demographic predictors.

\begin{tabular}{lcccc}
\hline & Estimate & Std. Error & $\mathbf{t}$ Value & $\operatorname{Pr}(>|\mathbf{t}| \mathbf{)}$ \\
\hline (Intercept) & -0.0077 & 0.2751 & -0.0279 & 0.9779 \\
$\mathrm{~A}$ & -1.5889 & 0.8470 & -1.8758 & 0.0688 \\
$\mathrm{H}$ & -0.7187 & 0.8470 & -0.8485 & 0.4018 \\
gender.1 & 0.4214 & 0.3313 & 1.2721 & 0.2115 \\
age.1 & -0.0189 & 0.0923 & -0.2045 & 0.8391 \\
Household.1 & -0.0838 & 0.1429 & -0.5864 & 0.5613 \\
Education.1 & 0.0525 & 0.0906 & 0.5797 & 0.5657 \\
Employment.1 & 0.1299 & 0.1134 & 1.1457 & 0.2595 \\
Income.1 & 0.3690 & 0.0809 & 4.5635 & 0.0001 \\
gender.3 & -0.0629 & 0.3313 & -0.1898 & 0.8505 \\
age.3 & -0.0002 & 0.0923 & -0.0026 & 0.9979 \\
Household.3 & -0.0056 & 0.1429 & -0.0390 & 0.9691 \\
Education.3 & 0.0876 & 0.0906 & 0.9665 & 0.3402 \\
Employment.3 & 0.1934 & 0.1134 & 1.7057 & 0.0967 \\
Income.3 & 0.2117 & 0.0809 & 2.6182 & 0.0128 \\
$R^{2}$ & 0.6877 & & & \\
F-statistics & 5.6620 & & & \\
$p$-value & 0.00001 & & & \\
\hline
\end{tabular}

Taking the parameters from Table 3 into two groups, the models (21) for the two shares can be explicitly written as follows:

$$
\begin{gathered}
\alpha_{A}=\exp (-1.5889+0.4214 \text { Gender }-0.0189 \text { Age }-0.0838 \text { Household } \\
+0.0525 \text { Education }+0.1299 \text { Employment } \\
+0.3690 \text { Income }), \\
\alpha_{H}=\exp (-0.7187-0.0629 \text { Gender }-0.0002 \text { Age }-0.0056 \text { Household } \\
+0.0876 \text { Education }+0.1934 \text { Employment } \\
+0.2117 \text { Income }) .
\end{gathered}
$$

The models (25) for priorities can be used for prediction of the preference in the multinomial shares (18), with one set of parameters put to zero, so the shares of all three items correspond to the normalization as in the relation (13):

$$
p_{A}=\frac{\alpha_{A}}{1+\alpha_{A}+\alpha_{H}}, p_{E}=\frac{1}{1+\alpha_{A}+\alpha_{H}}, p_{H}=\frac{\alpha_{H}}{1+\alpha_{A}+\alpha_{H}} .
$$

With any combination of the predictors' values, we can easily find the priorities (25) and estimate the shares of preferences (26). A useful way of investigating how the priorities depend on a specific predictor is as follows: holding the values of all other predictors on their mean level and changing the values of the predictor of interest, we can profile all the shares (26) by this predictor. An example of such profiling of priorities by the income is presented in Figure 1.

Figure 1 shows that with growing income the preference or the flavor A increases, of the flavor $\mathrm{E}$ decreases, and of the flavor $\mathrm{H}$ stays about at the same level. The flavor A, the least preferable for a small income, becomes the most preferred for the higher income groups, overcoming even the item $\mathrm{H}$ at the income of approximately $80 \mathrm{~K}$.

If to build the regression models (14)-(16) without the auxiliary predictors only by their intercepts, in other words taking only the indicator predictors from columns $\mathrm{A}$ and $\mathrm{H}$ in Table 2, the predicted constant priorities get the values:

$$
p_{A}=0.3348, p_{E}=0.1937, p_{H}=0.4715
$$

The graphs in Figure 1 can be considered relatively the corresponded to each item constant mean level (27). These values coincide with geometric means by the elements in 
each row of all AHP matrices of (22) kind, which is the known way of priority estimation in multi-person decision making.

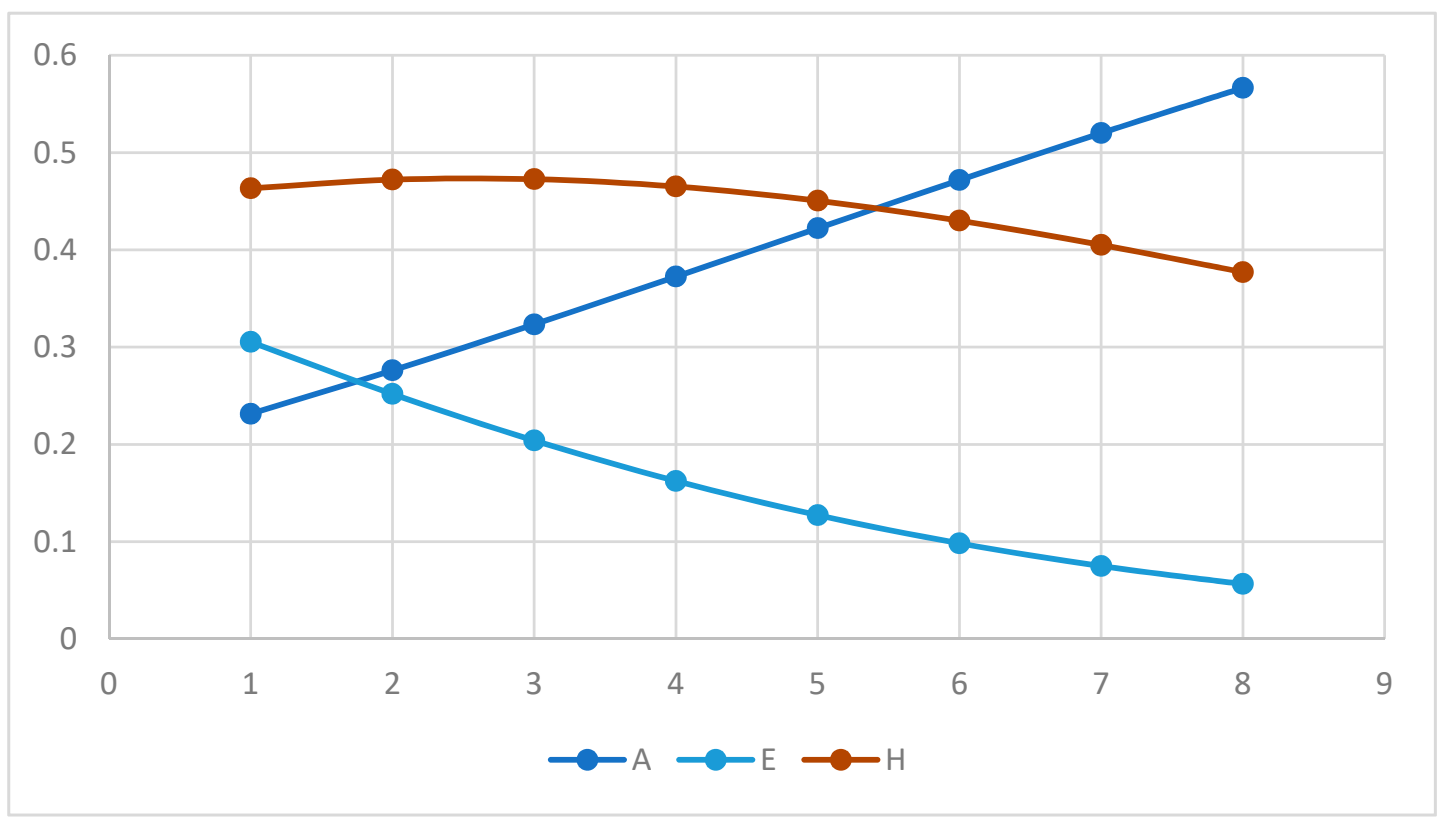

Figure 1. AHP three flavors priorities profiled by income.

For all $n=8$ flavors in this dataset, there are 28 pair ratios, and with $N=17$ respondents the relation (23) yields 476 observation rows in a matrix like in Table 2, but with 7 sets of the $M=6$ auxiliary demographic predictors (because one set is taken as the basic with its parameters put to zero). The total number of parameters for estimation becomes $(n-1) \cdot(M+1)+1=50$. The inequality (24) is approximately $68>7$, so the number of observations is near ten times more than the number of parameters in the regression and it can be built without problems. Then similarly to the Equations (25a)-(27) the priority results can be obtained by all eight flavors.

A simple estimation of priorities mentioned in relation to the models (21) can be also applied for each flavor, yielding parameters presented in Table 4 . The row below the predictors shows the coefficient of multiple determination $R^{2}$ serving as a characteristic of the fit in a model. The obtained models are reasonably good, and a lower value of $R^{2}$ for the flavor $\mathrm{H}$ means that this priority keeps a stable value across the varying predictors. The last row in Table 4 presents the shares of the mean preferences and these values define the vector of priorities by all seventeen respondents averaged. We see that in general the flavor $\mathrm{H}$ is of the higher preference, the next are the flavors $\mathrm{C}$ and $\mathrm{A}$, then go $\mathrm{F}, \mathrm{G}$, and $\mathrm{B}$, and the least preferred are $\mathrm{D}$ and $\mathrm{E}$.

A more detail investigation on the priorities of the flavors depending on the specific of the respondents can be found in profiling by the demographic features. Changing one predictor due to its values from Table 1, while keeping all the others at their mean levels produces the dependence of the priorities by the particular predictor. Figure 2 demonstrates behavior of the preferences by the income predictor. We see a drastic increase from the worst to the best positions for the $\mathrm{B}$ and $\mathrm{D}$ flavors, some improvement in A position, drop in levels of four other flavors, and a concave behavior of the $\mathrm{H}$ flavor with the maximum reached near the level of income about 50-74 K. Although there is no data in Table 1 for the customers with income of 125-149 K, the prediction for those can be made by the estimated priority shares as well. In general, the behavior of the three flavors $\mathrm{A}, \mathrm{E}$, and $\mathrm{H}$ shown in Figure 2 repeats their profiling in separate consideration presented in Figure 1, so the results of both approaches support each other. 
Table 4. Parameters of the exponential models for priority by demographics.

\begin{tabular}{ccccccccc}
\hline Predictors & Model A & Model B & Model C & Model D & Model E & Model F & Model G & Model H \\
\hline (Intercept) & 0.915 & 1.724 & 1.232 & 1.305 & 2.461 & 1.263 & -0.214 & 1.919 \\
gender & 0.463 & -0.563 & 0.184 & 0.190 & 0.082 & -0.257 & -0.035 & -0.063 \\
age & -0.003 & -0.070 & 0.027 & -0.070 & 0.018 & 0.003 & 0.098 & -0.003 \\
Household & 0.014 & -0.150 & 0.096 & -0.390 & 0.075 & 0.177 & 0.132 & 0.046 \\
Education & -0.109 & 0.007 & 0.026 & -0.028 & -0.154 & 0.109 & 0.213 & -0.064 \\
Employment & -0.055 & 0.045 & -0.027 & 0.094 & -0.198 & 0.062 & 0.045 & 0.033 \\
Income & 0.162 & 0.287 & -0.126 & 0.262 & -0.204 & -0.201 & -0.193 & 0.013 \\
$R^{2}$ & 0.402 & 0.463 & 0.379 & 0.799 & 0.612 & 0.491 & 0.534 & 0.152 \\
Mean share & 0.137 & 0.113 & 0.141 & 0.105 & 0.082 & 0.129 & 0.123 & 0.172 \\
\hline
\end{tabular}

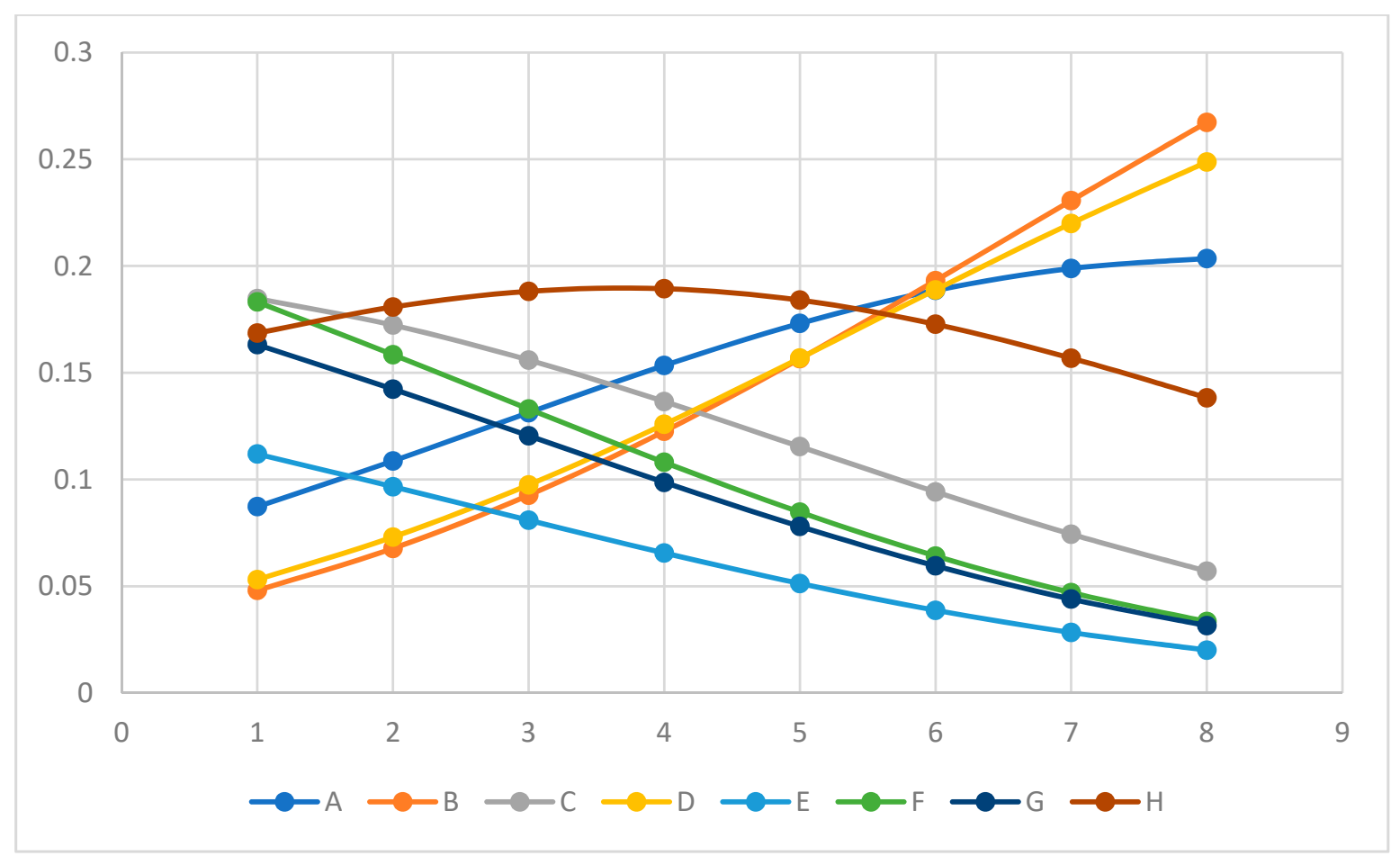

Figure 2. AHP eight flavors priorities profiled by income.

\section{Summary}

The paper describes the Analytic Hierarchy Process (AHP)—one of the most popular method for solving various problems of multiple-criteria decision making. AHP vectors of priority are calculated using pairwise comparison matrices to find preferences among different alternatives, and the elements of these vectors are commonly presented by the constant values. Estimations of the elicited data quality, particularly, the commonly observed non-transitivity between the pairwise ratios, is usually added to any AHP analysis, including such characteristics as the so-called consistency index and consistency ratio, compatibility measures, and precision criteria [13]. In a case of poor quality, a new data eliciting could be needed, and the robust estimations of AHP priorities can be applied as well [26].

For multiple respondents with their matrices of paired comparisons elicited in different conditions with varied characteristics of measurements, the priority vectors can be built not as constants but as functions of additional auxiliary predictors, for example, the respondents' demographic and socio-economic properties. This work explores new possibilities of building the priority vectors as functions found via regression modeling. 
The varying priorities can be predicted for individual respondents, profiled by each predictor, forecasted in time, studied by the predictor importance, and estimated by the characteristics of significance and quality known in the regression modeling. Numerical results show that the proposed approach reveals useful features of priorities behavior which extends AHP abilities to numerous multiple-criteria decision making problems. These new investigations on the priorities of choice among many alternatives can help to managers to introduce specific advertising and to develop focused programs for promotion of products to the individual customers of various demographics, socio-economical, cultural, and other possible characteristics measured by the auxiliary predictors.

The considered methods are useful for segmentation of the respondents with similar features and finding optimum managerial solutions specific for each segment, helping to increase customer satisfaction, their retention, and loyalty to the promoted brands or products. The obtained results are encouraging - they show that a specific set of conditions and prescriptions can be found for each respondent, or customer, that can be very valuable for managerial decisions, for example, in marketing and advertising research, and other areas of applied psycho-sociological statistical analysis.

Funding: This research received no external funding.

Institutional Review Board Statement: Not applicable.

Informed Consent Statement: Not applicable.

Data Availability Statement: Not applicable.

Acknowledgments: I am very grateful to three reviewers and Co-Editor of Stats Stéphane Mussard for useful comments and suggestions which improved the paper.

Conflicts of Interest: The author declares no conflict of interest.

\section{References}

1. Saaty, T.L. A scaling method for priorities in hierarchical structures. J. Math. Psychol. 1977, 15, 234-281. [CrossRef]

2. Saaty, T.L. The Analytic Hierarchy Process; McGraw-Hill: New York, NY, USA, 1980.

3. Saaty, T.L. Fundamentals of Decision Making and Priority Theory with the Analytic Hierarchy Process; RWS Publications: Pittsburgh, PA, USA, 1994.

4. Saaty, T.L. Decision Making with Dependence and Feedback: The Analytic Network Process; RWS Publications: Pittsburgh, PA, USA, 1996.

5. Saaty, T.L. Theory and Applications of the Analytic Network Process: Decision Making with Benefits, Opportunities, Costs, and Risks; RWS Publications: Pittsburgh, PA, USA, 2005.

6. Saaty, T.L.; Vargas, L.G. Comparison of eigenvalue, logarithmic least squares and least squares methods in estimating ratios. Math. Model. 1984, 5, 309-324. [CrossRef]

7. Saaty, T.L.; Vargas, L.G. Decision Making in Economic, Political, Social and Technological Environment with the Analytic Hierarchy Process; RWS Publications: Pittsburgh, PA, USA, 1994.

8. Saaty, T.L.; Kearns, K.P. Analytical Planning; Pergamon Press: New York, NY, USA, 1985.

9. Saaty, T.L.; Peniwati, K. Group Decision-Making: Drawing Out and Reconciling Differences; RWS Publications: Pittsburgh, PA, USA, 2007.

10. Whitaker, R. Validation examples of the Analytic Hierarchy Process and Analytic Network Process. Math. Comput. Model. 2007, 46, 840-859. [CrossRef]

11. Lootsma, F. Multi-Criteria Decision Analysis via Ratio and Difference Judgement; Kluwer Academic Publishers: London, UK, 1999.

12. Lipovetsky, S. Global Priority Estimation in Multiperson Decision Making. J. Optim. Theory Appl. 2009, 140, 77-91. [CrossRef]

13. Lipovetsky, S. Priority vector estimation: Consistency, compatibility, precision. Int. J. Anal. Hierarchy Process. 2020, 12, 577-591.

14. Lipovetsky, S. Personalized Key Drivers for Individual Responses in Regression Modeling. Int. J. Risk Conting. Manag. 2020, 9 , 15-30. [CrossRef]

15. Lipovetsky, S.; Tishler, A. Interval estimation of priorities in the AHP. Eur. J. Oper. Res. 1999, 114, 153-164. [CrossRef]

16. Lipovetsky, S. The synthetic hierarchy method: An optimizing approach to obtaining priorities in the AHP. Eur. J. Oper. Res. 1996, 93, 550-564. [CrossRef]

17. Lipovetsky, S. Conditional and multinomial logits as binary logit regressions. Adv. Adapt. Data Anal. 2011, 3, 309-324. [CrossRef]

18. Lipovetsky, S. Quantum paradigm of probability amplitude and complex utility in entangled discrete choice modeling. J. Choice Model. 2018, 27, 62-73. [CrossRef] 
19. Lipovetsky, S. Express analysis for prioritization: Best-Worst Scaling alteration to System 1. J. Manag. Anal. $2019,7,12-27$. [CrossRef]

20. Demidenko, E. Advanced Statistics with Applications in R; Wiley: Hoboken, NJ, USA, 2020.

21. Ismay, C.; Kim, A.Y. Statistical Inference via Data Science: A ModernDive into R and the Tidyverse; Taylor \& Francis: Boca Raton, FL, USA, 2020.

22. Irizarry, R.A. Introduction to Data Science: Data Analysis and Prediction Algorithms with R; Taylor \& Francis: Boca Raton, FL, USA, 2020.

23. Mair, P. Modern Psychometrics with R; Springer: Cham, Switzerland, 2018.

24. Lipovetsky, S.; Conklin, M. Analysis of regression in game theory approach. Appl. Stoch. Models Bus. Ind. 2001, 17, 319-330. [CrossRef]

25. Lipovetsky, S.; Conklin, M. Predictor relative importance and matching regression parameters. J. Appl. Stat. 2015, 42, 1017-1031. [CrossRef]

26. Lipovetsky, S.; Conklin, W.M. Robust Estimation of Priorities in the AHP. Eur. J. Oper. Res. 2002, 137, 110-122. [CrossRef] 